\title{
La personnalisation de l'impersonnel. Réflexion autour du commerce de l'igname à Cotonou, Bénin
}

Jean Adanguidi

\section{OpenEdition}

\section{Journals}

Édition électronique

URL : http://journals.openedition.org/apad/438

DOI : 10.4000/apad.438

ISSN : 1950-6929

Éditeur

LIT Verlag

Édition imprimée

Date de publication : 1 juin 2000

\section{Référence électronique}

Jean Adanguidi, «La personnalisation de l'impersonnel. Réflexion autour du commerce de l'igname à Cotonou, Bénin », Bulletin de l'APAD [En ligne], 19 | 2000, mis en ligne le 24 juillet 2006, consulté le 08 septembre 2020. URL : http://journals.openedition.org/apad/438 ; DOI : https://doi.org/10.4000/apad. 438

Ce document a été généré automatiquement le 8 septembre 2020.

Bulletin de l'APAD 


\title{
La personnalisation de l'impersonnel. Réflexion autour du commerce de l'igname à Cotonou, Bénin ${ }^{1}$
}

\author{
Jean Adanguidi
}

1 La chute du commerce de l'igname en direction du Nigeria vers la fin des années 1980, suite aux difficultés économiques de ce pays, avait entraîné le repli des commerçants béninois sur les marchés intérieurs. Le sud du pays plus densément peuplé est devenu dès lors un excellent débouché pour la production nationale. Il en a résulté un développement de la filière d'approvisionnement du Sud à partir de 1990. La caractéristique principale de cette filière, est sa structuration en circuits commerciaux. Nous en avons recensé trois principaux. Chacun d'eux gère des aires de production bien déterminées (cf. Graphique 1 et 2) :

- le circuit fon, animé par les commerçants du groupe ethnique Fon, gère la production du Nord des départements du Zou et de l'Ouémé. Il approvisionne le marché en igname fraîche ;

- le circuit bariba-tchabè, animé par les commerçants des groupes ethniques Bariba et Tchabè, gère la production de la région de Tchaourou, du Nord de Savè et de l'Est de Ouessè. Il approvisionne le marché surtout en cossettes d'igname mais également en igname fraîche ${ }^{2}$

- le circuit dendi-tanéka, animé par les commerçants des groupes ethniques Dendi et Tanéka. Il gère la production des régions de Djougou et de Copargo et approvisionne le marché principalement en igname fraîche.

2 Les principaux acteurs de ces différents circuits orientent l'essentiel de leurs activités sur la ville de Cotonou ${ }^{3}$, qui est d'ailleurs le plus grand centre de consommation du pays. Ce point de convergence des activités constitue le lieu de déploiement des stratégies d'acteurs, dans un espace marchand que nous pouvons assimiler à une arène, concept définit par Olivier de Sardan (1997) comme étant le "lieu de confrontations concrètes d'acteurs sociaux en interaction autours d'enjeux communs". Pour le cas d'espèce, l'enjeu principal est la conquête du gain de productivité. Cette conquête met 
en jeu des acteurs dotés de capitaux divers (économique, social, symbolique), donc ayant des pouvoirs de négociation différents. Bien que ces pouvoirs soient souvent évoqués dans les échanges marchands, les mécanismes de leur exercice sont généralement peu explicités.

La présente étude se propose de faire une analyse socio-anthropologique du commerce de gros dans la ville de Cotonou. Elle a pour but de montrer comment dans un univers aussi cosmopolite que celui de la distribution de l'igname à Cotonou, l'économique s'enchâsse dans le social.

L'organisation de la vente de gros au niveau du circuit fonPrincipaux acteurs

Deux catégories d'acteurs interviennent dans la distribution de l'igname à Cotonou : les grossistes et les détaillantes.

Les grossistes sont en majorité des hommes. Les femmes y sont faiblement représentées. Mais quand on voit l'importance des transactions au cours de l'année, on s'aperçoit que le plus grand grossiste de ce circuit, qui est en même temps le plus grand commerçant d'igname de tous les circuits qui approvisionnent le Sud-Bénin, est une femme (Adanguidi 1999). La particularité de ce circuit est que tous les grossistes ne sont pas basés à Cotonou. Une partie de ceux-ci résident à Bohicon (y compris la plus grande grossiste) et quelques uns à Djidja.

Graphique 1 : Zone de production d'igname au Bénin

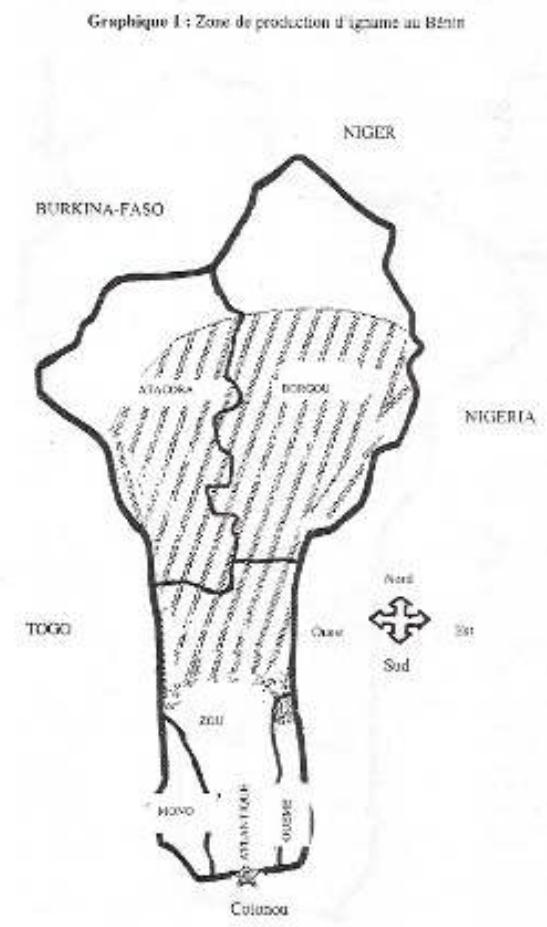

Voir carte en annexe

Graphique 2 : Les différents réseaux marchands 


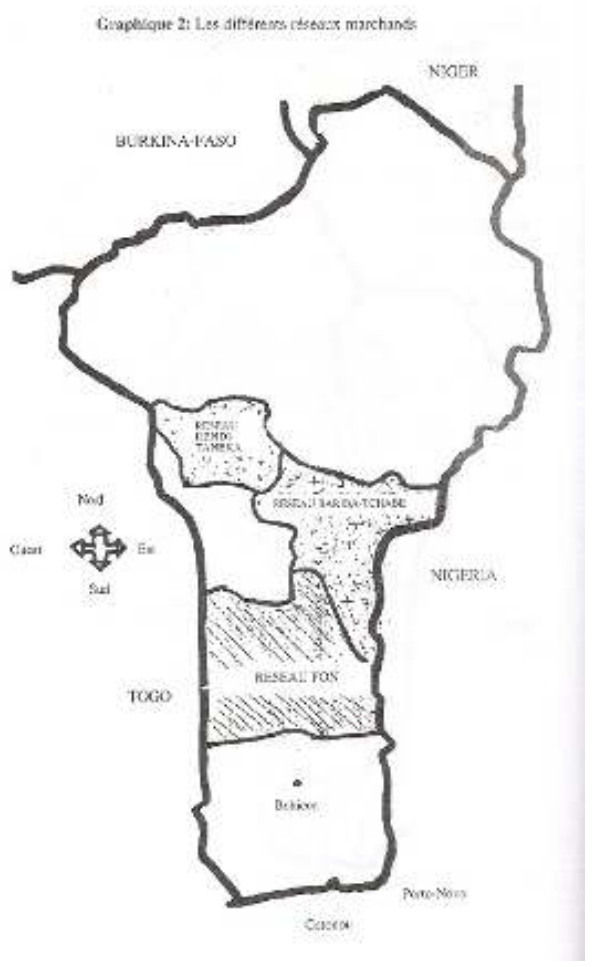

Voir carte en annexe

6 Les détaillantes appartiennent pour la plupart au groupe ethnique Fon. Celles appartenant aux groupes ethniques Bariba et Tchabè se ravitaillent rarement auprès des grossistes de ce circuit. Cette situation est liée aux habitudes commerciales et à la clientèle des différents groupes en présence. Les détaillantes Bariba et Tchabè ne sont pas habituées à l'achat et la revente à la bascule pratiqués par les Fon et ceci contrairement aux grossistes de ces deux groupes ethniques qui utilisent parfois la bascule pour se ravitailler dans les zones de production. Pour le cas de Tchatchou par exemple, la grossiste n'est pas tenue de savoir manipuler la bascule. Elle a la possibilité de recourir aux peseurs qui en ont parfaitement la maîtrise. La seconde raison avancée est d'ordre commercial. Chez les détaillantes Bariba et Tchabè, la clientèle constituée en majorité de femmes spécialistes de plats cuisinés et originaires du nord, serait surtout intéressée par l'igname en provenance de leur région, igname plus adapté au pilage et aux frites. Une détaillante Tchabè nous donnait comme exemple, la variété d'igname du nom de djougoulévi vendue par les grossistes du circuit dendi-tanéka. Selon cette dernière, la variété gnidou étant moins chère, ceux qui aiment la quantité achètent chez les Fon pendant cette période. Ceux qui aiment la qualité achèteraient chez les détaillantes du Nord qui vendent djougoulévi. Personnellement, nous n'avons pas d'arguments solides sur les qualités organoleptiques des différentes variétés, pour prendre position sur une pareille assertion. Nous avons observé cependant au sein de chaque circuit, la pratique de prix différenciés en fonction de la qualité du produit. Dans le circuit fon par exemple, la variété lamboko coûte plus cher que les autres parce qu'elle est mieux adapté au pilage.

Principaux enjeux

7 L'enjeu des différents acteurs est la conquête du gain de productivité. Cette conquête dépend en grande partie du poids de chaque acteur dans le marché. Les principaux 
enjeux se jouent autour de l'accès au moyen de transport, l'accès à la place de marché et l'accès à la clientèle.

8 L'importance du capital commercial détermine le poids du grossiste dans la profession. Une composante importante de ce capital, est le transport, dont le coût et les modalités d'accès sont déterminants dans l'activité des commerçants (Clark, 1994 : 149; Godart, 1985). Pour les grossistes de ce circuit, l'accès aux camions de transport est nécessaire pour le succès dans la profession, car l'achat de la marchandise se fait surtout à la ferme. La maîtrise du transport constitue une source de pouvoir chez les commerçants. La plupart des grossistes de ce circuit possèdent un camion. Ceux qui n'en ont pas ont recours à la location. Ce qui les désavantage et ceci pour deux raisons.

D'abord ils n'ont pas une grande flexibilité. C'est quand les propriétaires de camions n'ont pas programmé de voyage, qu'ils les louent à ceux qui n'en possèdent pas. On comprend dès lors que quand le marché est intéressant, c'est-à-dire que la demande augmente, les non-propriétaires de camion ne puissent convenablement en tirer profit. Cette situation est aggravée ces dernières années par le développement de la vente directe sur le parc automobile à partir des camions. Cette stratégie, développée par la plus grande grossiste d'igname du Sud-Bénin, de surcroît propriétaire de plusieurs camions, a été suivie par les autres commerçants qui possèdent également des camions. La tentative d'opposition des commerçants non-propriétaires de camion de couper cours à cette pratique qui les pénalisent s'est soldée par un échec. Or dans le même temps, il y avait eu vent que ceux qui vendent dans les magasins truquent leurs bascules, à telle enseigne que les détaillants préfèrent s'adresser à ceux qui garent leurs camions sur le parc automobile et dont les bascules étaient supposées être plus transparentes. Cette innovation a fait chuter les grossistes moins nantis à l'exception de ceux qui disposaient d'une clientèle très fidèle. Certains étaient alors obligés d'emboîter le pas des propriétaires de camions.

Ensuite, la mobilisation du camion leur revient cher. Le carillon est négocié sur une base journalière. Cela veut dire que plus vous immobilisez le camion plus vous payez. Il faut donc avoir assez de moyens pour louer un camion à garer sur le parc automobile parfois jusqu'à quatre jours, afin d'écouler la marchandise. Pour les propriétaires de camion, le problème d'immobilisation du carillon se pose avec beaucoup moins d'acuité. D'ailleurs, certains n'hésitent pas à diminuer le prix de leur marchandise afin de vite l'écouler. Par exemple, lorsque le prix du panier est à 11000 FCFA, ils cèdent la marchandise au prix de 10000 FCFA à ceux ${ }^{4}$ qui ont de magasins sur place dans le marché.

11 L'accès à la place de marché était un véritable enjeu au moment où la vente directe à partir des camions n'était pas développée. La marchandise était autrefois déchargée dans les magasins puis écoulée progressivement. La capacité de stockage de la marchandise était pour le commerçant une source de pouvoir. La plupart des grossistes de ce circuit, ont au moins un magasin dans le marché. Bien qu'il ne soit pas possible de garder l'igname sur une longue période, le stockage a l'avantage de permettre aux commerçants de contrôler quelque peu l'offre ou tout au moins les fluctuations de prix qu'il juge défavorable sur une courte période. Le magasin en même temps qu'il sert de lieu de stockage de la marchandise, sert également pour certains comme lieu de couchage. Le nombre de magasins détenus par un commerçant dépend de son degré de maitrise des circuits officiels (contrôlé par la SOGEMA ${ }^{5}$ ) et non-officiel d'attribution des magasins dans le marché. D'ailleurs, les commerçants le disent explicitement à qui 
veut les entendre : pour obtenir un magasin de la SOGEMA, il faut avoir non seulement de l'argent mais surtout des relations. Le processus d'attribution des magasins apparait donc comme un véritable terrain de négociation où se déploient des actes de corruption de toute nature. On comprend dès lors, l'inégale répartition des magasins de vente dans le marché dantokpa. Des commerçants ont pu donc avoir non seulement des magasins pour eux-mêmes, mais aussi pour leurs femmes, leurs enfants alors que dans le même temps, certains cherchent un seul et n'en trouvent pas. Il y a même un micro-business développé autour de ces magasins. Ceux qui n'arrivent pas à exploiter le ou les magasins qu'on leur a attribués, les mettent en location à des prix élevés. Par exemple, le coût officiel de location d'un magasin de la SOGEMA est de 1500 FCFA par mois. Mais dans le circuit non-officiel, cela se fait à 6000 ou 7000 FCFA le mois. Finalement, le développement ces dernières années de la vente directe à partir des camions, en même temps qu'il pénalise les grossistes non-propriétaires de camions, constitue un excellent recours pour les grossistes qui ne disposent pas de magasins à Cotonou, principalement ceux qui sont basés à Bohicon ou Djidja.

Le dernier et plus important enjeu de ce circuit, est l'accès à la clientèle. La constitution d'une clientèle plus ou moins fixe est fondamentale dans l'activité des grossistes. Chaque grossiste dispose d'une clientèle à qui il distribue sa marchandise dès son arrivée à Cotonou. Cette clientèle est constituée en majorité de détaillantes Fon. Les grossistes qui ont des parents dans la profession livrent une bonne partie de leur marchandise à ces derniers. Un grossiste nous expliquait d'ailleurs à cet effet, que chaque fois qu'il arrive à Cotonou avec une nouvelle marchandise il livre une bonne partie à sa femme, sa mère et ses sœurs qui font la revente au détail. Dans la pratique, la clientèle est d'abord constituée à partir du réseau familial. Elle s'élargit ensuite aux autres détaillantes Fon qui sont dans le marché.

La vente se fait à crédit remboursable après l'écoulement du produit par le client. Pendant la période où les commerçants vont se ravitailler sur le marché de Glazoué qui a lieu tous les mercredis, le remboursement se fait par le client la veille ou l'avant-veille du jour d'animation de ce marché, c'est-à-dire le lundi ou le mardi, afin de permettre au grossiste d'aller se ravitailler à nouveau. Mais pendant les périodes où les grossistes vont se ravitailler dans les fermes, le remboursement du crédit par le client n'intervient qu'après l'écoulement total du produit. Et à ce niveau, la force financière du commerçant, comme cela transparaît dans le récit suivant, joue un rôle déterminant.

"Mes clients fidèles qui achètent à crédit me remboursent le marché suivant ou dans une semaine au plus tard. Une fois remboursés, ils achètent à nouveau. Ceci explique qu'il faut avoir un double capital. Car tu vends à crédit le premier arrivage et tu repars à Glazoué pour amener un nouveau stock. Le client vient rembourser et achète à nouveau à crédit. Si tu n'as pas un double capital, tu es obligé d'attendre récupérer les fonds avant de repartir à Glazoué. Il arrive que le client ne rembourse pas. Certains fuient. D'autres reviennent acheter à crédit et remboursent progressivement. Certains cessent même d'arriver sur le marché et on passe les voir à la maison pour retirer l'argent. Mais que voulez-vous? On est obligé de continuer à vendre à crédit, si non plus vous gardez l'igname, plus les pertes (perte de poids et pourriture) augmentent."

Dans ce récit, nous voyons donc que, la vente à crédit en même temps qu'elle constitue une bonne stratégie de vente en période de pénurie comme d'abondance est également une stratégie de répartition du risque au sein de la profession. Car lorsque le commerçant livre rapidement sa marchandise à ses clients à crédit, la gestion de la 
perte de poids consécutive à une longue durée de stockage devient systématiquement l'affaire de la détaillante. Le crédit est donc un moyen de fidélisation de la clientèle. Un constat analogue a été fait par Clark (1994: 174) dans le marché central de Kumasi au Ghana.

En conclusion de cette partie, nous dirons donc que le degré de contrôle des moyens de commercialisation (transport, place de marché et clientèle), est déterminant pour la conquête de la part de marché. Il permet en outre de comprendre, les différentes pratiques de domination et de subordination entre les agents en présence et qui font de la place du marché une arène. La constitution d'une clientèle plus ou moins fixe a plusieurs avantages pour le commerçant.

- Elle lui permet de ne plus investir trop de temps dans la recherche de clients pour sa marchandise. Convaincre un nouveau client demande habituellement plus de temps et d'effort. Or pour un ancien client ou plus précisément un client fidèle, le travail préalable a été déjà fait.

- Elle lui permet également de réduire les risques liés à la profession. Pour un produit périssable comme l'igname, une telle option est intéressante en ce sens qu'elle permet de répartir les risques avec les autres acteurs de la filière. Le commerçant qui livre rapidement sa marchandise à ses clients n'aura plus à gérer seul, la perte de poids ou la pourriture des tubercules consécutive à une longue durée de stockage.

- Elle réduit également le risque de mévente. Car, le grossiste tient surtout compte de la taille de sa clientèle fidèle pour acheter la marchandise dans les zones de production.

- En somme, cette stratégie permet aux grossistes de réduire les coûts de transaction. La personnalisation des relations permet aux détaillantes d'accéder à la marchandise à crédit, ce qui leur donne la possibilité de se maintenir dans la profession sans disposer de fonds de roulement propre.

Organisation de la vente de gros au niveau du circuit Bariba- TchabèPrincipaux acteurs

Dans ce circuit, trois catégories d'acteurs interviennent dans la vente de gros ${ }^{6}$ à Cotonou : les grossistes, les magasiniers et le détaillantes.

Les grossistes sont essentiellement des femmes, basées non pas à Cotonou mais plutôt dans leurs régions d'origine (Tchaourou, Toui dans Savè, Kilibo et PK dans Ouessè). La plupart de ces commerçantes n'arrivent à Cotonou que pendant la campagne de commercialisation de l'igname dans leurs régions respectives. Contrairement aux commerçants Fon spécialisés dans le commerce des ignames fraîches, les commerçantes de ce circuit vendent aussi bien les ignames fraîches que les cossettes d'igname et quelquefois même le maïs. La vente des ignames fraîches se fait pendant la saison des pluies et une bonne partie de la saison sèche (variété kokoro). Par contre, la vente des cossettes d'igname intervient surtout pendant la saison sèche. $C^{\prime}$ 'est pour cela qu'une bonne partie de ces femmes sont actives dans la profession (vente de l'igname et de ses dérivés) toute l'année. L'effectif des commerçantes a connu une augmentation ces dernières années. Quand on analyse les histoires professionnelles de ces commerçantes, on se rend compte que la plupart étaient d'anciennes collectrices villageoises qui ont fini par se lancer dans le commerce de longue distance en direction de Cotonou. Pour le cas particulier des femmes de tchatchou que nous avons étudié, une commerçante nous a raconté ce qui suit :

"Le nombre de femmes qui font le commerce en direction de Cotonou a augmenté ces dernières années ; car ce que les femmes faisaient dans les villages, c'est-à-dire la collecte d'igname dans les campements peuls ne marchait plus comme autrefois. $\mathrm{Au}$ départ, quand on allait dans les campements peuls, on faisait 1000 à 1500 FCFA 
de bénéfice par jour. Aujourd'hui les Peuls ne se laissent plus faire comme avant si bien que difficilement on réalise un bénéfice de 300 à 400 FCFA par jour. Et pour trouver ce peu, il faudra se promener à longueur de journée et aller dans les fermes reculées pour trouver de marchandise à un prix abordable. C'est pour cela que beaucoup de femmes se sont lancées dans le commerce en direction de Cotonou qui d'ailleurs marche mieux. Comme certaines femmes ne peuvent pas rester les bras croisés à la maison, elles continuent ce travail de collecte dans le village. C'est pour cela que le nombre de collectrices aussi augmente de jour en jour." originaire du Niger. Ils ont un double statut :

- D'abord, ce sont des gérants de magasins de stockage de produits vivriers dans le marché. Chacun d'eux dispose d'un grand magasin. Ils perçoivent une commission de 200 FCFA par sac de marchandise déposé dans leur magasin.

- Ensuite, ce sont des courtiers commerciaux. Ils assurent la vente de marchandises aux commerçantes qui le désirent moyennant une rémunération de 200 FCFA par sac. Leur atout réside dans le fait qu'ils sont basés à cotonou (contrairement aux grossistes), connaissent les circuits de distribution des produits vivriers, et surtout parlent bien la langue Fon couramment utilisée sur le marché. Ils ont une bonne connaissance de la situation de l'offre et de la demande des di vers produits et sont aussi en contact permanent avec les détaillantes du marché.

Lajorité des détaillantes, pour leur part, appal1iennent aux groupes ethniques Bariba et Tchabè. Certaines détaillantes Fon se ravitaillent chez les grossistes de ce circuit lorsque le différentiel prix par rapport à la vente au panier pratiquée par les grossistes Fon est intéressant. Le mode de vente des grossistes de ce circuit varie en fonction de la grosseur des tubercules.

Organisation de la vente des ignames précoces

des ignames précoces au niveau de ce circuit a pris de l'importance à partir de 1994. Les grossistes vendent par tas de quatre tubercules aux détaillants. Car, il s'agit de gros tubercules qui ne se prêtent pas à la vente au sac. Même dans les zones de production, l'achat se fait au tas.

21 Dès l'arrivée de la commerçante, les détaillantes qui sont en rupture de stock, se regroupent par affinité pour acheter toute la marchandise qu'elles se partagent ensuite entre elles compte tenu de leurs besoins respectifs. Une fois l'opération terminée, le groupe se disloque. Ce sont donc en réalité des groupements de circonstance qui se créent juste au moment de la transaction avec la grossiste. Lorsqu'une autre commerçante arrive dans le marché avec sa marchandise, un autre groupement se crée. Dans le cas où plusieurs commerçantes arriveraient le même jour, plusieurs groupements se créent. Ces derniers se retrouvent dans une double situation d'oligopole et d'oligopsone. Car, les consommateurs et les restauratrices n'arrivent pas à accéder directement à la marchandise auprès des grossistes de ce circuit. Ils sont également les seuls qui vendent le produit aux consommateurs et aux restauratrices. La force de ces groupements qui se construisent et se disloquent semble diminuer ces dernières années. Car il y a quelques années, tous les groupements s'entendaient sur un prix de vente et négocient cela avec les grossistes. Mais avec l'augmentation de leur effectif, tous les groupements ne s'entendent plus sur un prix avant le démarrage de la transaction. Chaque groupement négocie directement avec la commerçante. 

circuit depuis 1998 est le recours à la vente à la bascule par certaines grossistes de Tchaourou afin de vite écouler la marchandise lorsque la quantité est importante. Les frais de location de la bascule auprès des commerçants Fon s'élèvent à 250 FCFA/panier et sont payés par l'acheteur. Les détaillantes Fon utilisent leur propre bascule ou celle de leurs parents. Ce phénomène est donc récent et résulterait d'un "effet de contagion" engendré par les commerçants Fon qui pratiquaient depuis plusieurs années la vente à la bascule. Une autre raison serait liée à la percée lente mais progressive de la bascule dans les pratiques commerciales de ces commerçants dans leurs régions d'origine. Dans le village Tchatchou (sous-préfecture de Tchaourou) que nous avons étudié, la bascule est un peu utilisée dans les transactions. Cette innovation s'oppose encore à la résistance des commerçants du circuit dendi-tanéka qui n'ont pas l'expérience de la bascule, même dans leur région d'origine.

Organisation de la vente des ignames tardives

La vente des ignames tardives, essentiellement de la variété kokoro, dont la commercialisation démarre en décembre, se fait par $\operatorname{sac}^{8}$. Contrairement aux grossistes Fon chez qui la vente se fait directement à partir des camions garés sur le parc automobile, les grossistes de ce circuit confient plutôt leurs marchandises aux magasiniers qui leur en assurent la vente. La principale raison évoquée par ces commerçantes est qu'elles ne résident pas à Cotonou, elles ne maîtrisent pas bien le circuit de distribution et que par ailleurs, rares sont celles d'entre elles qui comprennent la langue Fon utilisé sur le marché. Une commerçante explique :

"Je ne comprends pas la langue Fon parlée sur le marché. Comment puis-je communiquer avec les gens? Je suis obligée de confier ma marchandise aux magasiniers. Même si on comprend le Fon, cela ne suffit pas pour vendre soi-même. Car, les magasiniers connaissent bien le marché et savent comment vendre le produit."

Le recours aux magasiniers semble être la transposition d'une pratique locale à Cotonou. D'après les histoires professionnelles de quelques commerçantes originaires de Tchatchou que nous avons enquêtées, trois phases ont marqué le développement de ce circuit. Les plus anciennes qui se sont lancées dans le commerce de l'igname, il y a environ 20 ans, avaient orienté leurs activités (principalement la vente des cossettes d'igname) vers les villes du Nigeria (Saki et Souya). Il s'agissait, bien entendu, de ('époque du boom pétrolier nigérian. Mais cela n'avait duré qu'environ 5 ans. Avec les mesures d'austérité prises par le gouvernement nigérian à partir de 1983, le commerce n'était plus intéressant et les commerçantes ont plutôt orienté leurs activités vers la ville de Parakou situé à $25 \mathrm{~km}$ de leur localité. Le commerce vers cette ville était centré sur trois produits : l'igname fraîche, les cossettes et le maïs. Le stock de marchandise était confié aux magasiniers qui en assuraient la vente moyennant une commission (100 FCFA par sac vendu). Mais le grand problème qu'elles avaient dans ce marché était la difficulté de récupération des fonds. Car la vente se faisait essentiellement à crédit. A cela s'ajouterait le fait que le commerce de l'igname en direction de cette ville ne soit pas assez rentable. Ces deux principales raisons expliqueraient le replis de ces commerçantes sur le marché de Cotonou depuis le début des années 90. Même celles qui se sont lancées dans la profession il y a moins de 6 ans (le plus grand nombre d'ailleurs), ont préféré orienter leurs activités vers Cotonou où il y a moins de problèmes pour la récupération des fonds. 

marchandise, la plupart des commerçantes passent la nuit dans les magasins. Quand l'offre est abondante. la commerçante vend parfois à crédit à certaines détaillantes qu'elle connaît. Le magasinier peut aussi vendre à crédit à certaines détaillantes. Mais dans ces conditions, il se charge de la récupération des fonds. Généralement, la grossiste rentre en possession de ses fonds avant de reprendre le chemin de retour. Lorsqu'elle n'y parvient pas, elle fait la récupération lors de la tournée suivante. Quelquefois, le magasinier lui envoie l'argent par l'intermédiaire d'une commerçante de chez elle. Une stratégie des commerçantes afin de vite écouler la marchandise est le partage du stock aux deux magasiniers. Il arrive qu'une commerçante empêchée envoie son fils ou sa fille à Cotonou avec la marchandise. Dans ce cas, la commerçante remet une lettre à cc dernier ou cette dernière, lettre destinée au magasinier devant vendre la marchandise. Cela veut dire que le commerçant du nord n' a pas besoin de connaître ou $\mathrm{d}^{\prime}$ avoir de relations particulières avec les détaillantes. Ceci est contraire à la situation observée chez les grossistes Fon, où il $\mathrm{y}$ a une forte personnalisation des relations avec les détaillantes. Il découle donc que la non maittrise du circuit de distribution par la commerçante du Nord est compensée par le recours à un magasinier qui lui est basé sur le marché, connaît bien la situation de l'offre et de la demande et qui de surcroît est originaire du Nord, ce qui est perçu comme un gage de confiance. Pour les grossistes de ce circuit, le recours au service du magasinier est obligatoire.

Organisation de la vente de gros au niveau du circuit dendi-tanékaPrincipaux acteurs

Nous distinguerons ici également trois catégories d'acteurs: les grossistes, les demi-grossistes occasionnels, et des détaillantes.

es grossistes sont principalement des femmes originaires de Djougou et de Copargo ${ }^{9}$ Elles sont pour la plupart basées dans leurs régions d'origine. La vente des ignames fraîches est l'activité principale de ces femmes. Celles originaires de Copargo vendent secondairement l'arachide le moment venu ${ }^{10}$. Celles originaires de Djougou amènent parfois des cabris et un peu de cossettes d'igname ${ }^{11}$. Numériquement, celles de Djougou sont plus nombreuses, car elles étaient les toutes premières à commencer le commerce de l'igname en direction de Cotonou ${ }^{12}$. L'effectif des commerçantes de Copargo qui font le commerce de l'igname en direction de Cotonou a connu une augmentation ces dernières années. Jusqu'en 1989, il y avait seulement une seule commerçante de Copargo qui faisait ce commerce. En 1990 un couple s'est joint à elle et le nombre passa à trois. Ce n'est qu'à partir de 1995- 1996 que le nombre a réellement augmenté et aujourd'hui, on dénombre environ une quinzaine de commerçants de Copargo qui font le commerce de l'igname en direction de Cotonou ${ }^{13}$. Au nombre de ceux-ci, il y a une forte proportion de femmes. Il est possible que ce regain d'intérêt pour cette activité soit suscité par l'amélioration du réseau routier dans le département de l'Atacora pendant cette période : le bitumage de l'axe Natitingou-Copargo-Djougou puis de l'axe Djougou-Parakou a été achevé en 1997.

Les demi-grossistes occasionnels sont en majorité des Dendi, originaires de la région de Djougou. Certaines, et c'est d'ailleurs la minorité, proviennent d'autres régions du département de l'Atacora comme Kouandé, Alédjo et Sèmèrè. Contrairement aux grossistes, elles sont basées à Cotonou et travaillent comme vendeuses de produits vivriers au marché Dantokpa (vente de cossettes d'igname, d'arachide, etc.). Nous les avons désignées comme demi-grossistes occasionnelles parce que la vente de l'igname fraîche n'est pas leur activité principale, mais plutôt une activité temporaire qu'elles 
mènent pendant les quelques mois que dure la commercialisation de l'igname de ce circuit.

Elles sont les intermédiaires incontournables des grossistes.

Les détaillantes, du circuit bariba-tchabè, appartiennent aux groupes ethniques Tchabè et Bariba. Elles se ravitaillent auprès des demi-grossistes occasionnelles. Quelques détaillantes Fon se ravitaillent aussi auprès de ces demi-grossistes.

Organisation de la vente de gros à Cotonou

L'activité des grossistes de ce circuit s'étend sur 10 mois environ, de septembre jusqu'en juin. L'importance de celle-ci varie selon qu'il s'agit des gros ou des petits tubercules, les deux produits arrivant sur le marché à des périodes différentes.

2 Les ignames précoces sont récoltées pendant la saison des pluies et leur commercialisation s'étale sur les mois de septembre, octobre et novembre. Elles ne se conservent pas longtemps. Elles sont recherchées sur le marché frontalier de Malanville qui est surtout fréquenté par les commerçants nigériens. La plupart des commerçants de Copargo abandonnent au cours de cette période le commerce de longue distance en direction de Cotonou et se consacrent simplement au travail de collecte dans leur localité.

3 Le produit collecté est revendu aux femmes de Djougou qui font le commerce en direction de Malanville.

Les quelques commerçantes de ce circuit qui font le commerce de l'igname en direction de Cotonou durant cette période, sont celles originaires de Djougou. Les ignames tardives ${ }^{14}$ sont récoltées pendant la saison sèche et leur commercialisation se fait de décembre à mai-juin. La première qualité de ces ignames cultivées dans la région de Copargo, est qu'elles peuvent être conservées pendant plusieurs mois ${ }^{15}$. De plus, elles sont recherchées pour leur qualité, car elles sont mieux adaptés au pilage et aux frites. Le commerce des ignames tardives en direction de Cotonou devient intensif à partir de février, et ce jusqu'à la sortie des nouvelles ignames ${ }^{16}$. La pratique des grossistes de ce circuit se démarque de celle des circuits précédents, en ce sens qu'il ne s'agit point de la vente directe de la marchandise aux détaillantes ou de la vente par l'entremise du magasinier ${ }^{17}$. Tout comme les grossistes du circuit bariba-dendi, elles ne maîtrisent pas la langue fon parlée dans le marché et ne connaissent pas les circuits de distribution de Cotonou. Pour contourner cette difficulté, ils vendent leurs marchandises en gros aux vendeuses de produits vivriers, originaires du département de l'Atacora (principalement de Djougou) et qui sont installées à Cotonou depuis plusieurs années. Chaque grossiste a sa clientèle de demi-grossistes à qui elle livre sa marchandise une fois arrivée à Cotonou. Ces dernières revendent ensuite la marchandise aux détaillantes. Une commerçante raconte :

"Arrivée à Cotonou, nous vendons notre marchandise par tas. Nous ne vendons pas à la bascule, ni au sac. Nous avons acheté par tas chez nous et nous revendons par tas. Dans la pratique, on fait un tas et puis on discute son prix avec nos clientes ${ }^{18}$. Une fois un compromis trouvé, la cliente compte le nombre de tas du stock et on multiplie par le prix unitaire. Lorsque les tubercules ne sont pas uniformes, on sépare les gros des petits et puis on compte chaque lot après avoir discuté du prix d'achat du tas. Par exemple si le tas de petits tubercules est vendu à 1200 FCFA, celui des gros tubercules peut être vendu à 2000 FCFA. Une seule cliente peut ramasser 200 tas. J'ai trois clientes à Cotonou : deux sont des Dendi originaires de Djougou et la troisième est une Bariba de Kouandé. Ce sont nos clientes qui vendent l'igname aux détaillantes y compris les Fon ; car, nous sommes des étrangers, nous 
ne les connaissons pas et nous ne savons pas non plus où ils habitent. Or les femmes Fon achètent surtout à crédit; comment pouvons-nous récupérer l'argent ? C'est nos clientes qui sont sur place qui leur vendent parfois à crédit et prennent l'argent plus tard". manifestation des rapports de confiance dans les transactions. Les grossistes ${ }^{21}$ livrent la marchandise à leurs clientes à crédit sans que le nom et la quantité achetée par cette dernière ne soient mentionnés sur un papier comme cela se fait chez les grossistes Fon. Parfois la grossiste retourne au Nord et la cliente lui envoie l'argent par l'intermédiaire d'une de ses collèges. La grossiste peut aussi envoyer de la marchandise à ses clientes par l'intermédiaire d'une collègue qui se rend à Cotonou. Une commerçante nous a expliqué le mécanisme :

"Tu peux rester à Copargo et confier des sacs (10 à 20 sacs) à une amie commerçante qui descend sur Cotonou pour tes clientes. Tu lui indiques à quel prix vendre le produit. Si arrivée à Cotonou, elle constate que le prix a chuté ou augmenté, elle t'informe à son retour. Elle te ramène ton argent si elle parvient à récupérer cela avant de revenir au village, etc. Cette dame vient de m'amener de l'argent. C'est une de mes clientes de Cotonou qui m'a envoyé l'argent. C'est une pratique assez répandue ici et qui témoigne, contrairement à ce qu'on pourrait imaginer, de la bonne collaboration qu'il y a entre les commerçants de notre localité. Nous nous connaissons bien et nous ne nous trahissons pas. Ce n'est pas comme au sud."

Il est même possible à une cliente de diminuer le prix de vente proposé par la grossiste qui lui envoie sa marchandise. Une demi-grossiste raconte :

"J'ai des commerçantes à Copargo qui m'envoient de marchandise avec une lettre qu'on me remet. Dans la lettre, elles précisent le nombre de tas qu'il y a dans le camion et proposent le prix de vente du tas. On modifie parfois le prix. Par exemple, si elles veulent vendre le tas à 1500 FCFA, elles proposent 1800 FCFA. Compte tenu de ma connaissance du marché, je sais à quel prix acheter la marchandise pour que cela les arrange et m'arrange aussi. Je peux diminuer le prix. Dans ce cas, les autres commerçantes de chez elles qui sont dans le marché, vont les informer à leur retour du changement intervenu."

La personnalisation des relations est poussée dans ce circuit. Lorsqu'une grossiste arrive avec sa marchandise dans le marché et ne trouvent pas ses clientes, elle les attend jusqu'à ce qu'elle arrivent. L'attente peut durer des heures et même parfois un à deux jours. La conséquence de cette situation est que les autres détaillantes ne peuvent accéder directement à la marchandise. Elles ne manquent d'ailleurs pas de se plaindre. L'une d'entre elles raconte :

"Ces commerçantes de Djougou ou de Copargo ne vendent leur marchandise qu'à leurs sœurs qui sont dans le marché. Quand elles arrivent et ne les trouvent pas, elles les attendent jusqu'à leur arrivée avant de commencer par vendre leur marchandise. C'est à partir de ce moment que nous achetons auprès des 
demi-grossistes. Elles nous revendent cela en gros mais un peu plus cher. Par exemple, si elles avaient acheté le tas à 500 FCFA, elles revendent cela à 600 FCFA. Pour l'igname de Copargo, on ne se lève pas pour acheter directement. C'est impossible. Moi je parviens parfois à le faire chez certaines commerçantes parce que je suis maternellement du Nord, j'ai grandi là-bas et je comprends leur langue. En dehors de moi, toutes les autres détaillantes achètent auprès des demi-grossistes. Ils vendent donc en priorité aux gens de leurs régions. C'est une question de solidarité régionale. Il y a parfois exception pour les gens qui arrivent voir un parent à Cotonou et qui amènent une petite quantité d'igname dans leur bagage. Comme ils n'ont pas de clientes, ils vendent à n'importe qui."

Les demi-grossistes de ce circuit jouent à peu près le même rôle que les magasiniers du circuit bariba-tchabè. La seule différence se trouve ici au niveau de leur mode de rémunération : le magasinier perçoit une commission alors que la femme intermédiaire se définit même sa rémunération qu'elle répercute sur le prix de revente de la marchandise.

Conclusion

Dans le circuit fon, le pouvoir de négociation du grossiste dépend de sa capacité à mobiliser les ressources. L'accès à ces ressources constitue un enjeu important. Par contre, dans les deux autres circuits, la situation est différente, car ces ressources ne constituent plus un enjeu. Les grossistes du nord n'ont pas besoin de disposer d'un camion pour mieux percer dans la profession. Ils résident dans les zones de production. Ils ont la possibilité d'avoir recours aux minibus, aux bâchées ou aux Jeeps pour le ramassage de leur marchandise des fermes jusqu'au bord d'une grande voie de communication afin d'emprunter les camions ou les titans en partance pour Cotonou. De plus, ils n'ont pas besoin de disposer d'une place de marché ou de chercher à conquérir une part de marché (clientèle), car le recours aux magasiniers et aux demi-grossistes leur permet d'écouler leur marchandise.

41 La forte personnalisation des relations a été observée dans tous les circuits de vente de gros à Cotonou. Elle s'opère sur une base régionalo-ethnique. Dans le circuit fon, cela se manifeste entre les grossistes et les détaillantes. Au niveau du circuit bariba-dendi, cela s'observe entre les grossistes et les magasiniers. Dans le circuit dendi-tanéka, la personnalisation des relations s'observe entre les grossistes et les demi-grossistes. Dans cette personnalisation des relations, les différents acteurs n'ont pas les mêmes pouvoirs de négociation. Au niveau du circuit fon, le pouvoir de négociation est fonction du degré d'accès aux ressources. Par contre, dans les deux autres circuits, c'est surtout la fonction de chaque agent qui est déterminante. Malgré l'existence de ce pouvoir différencié, la relation entre les principaux acteurs est surtout une relation de partenariat et s'oppose donc à la relation patron-client ou employeur-employé qui caractérise les réseaux clientélistes.

Dans le circuit fon, la vente de l'igname est pratiquement une entreprise familiale où sont impliqués l'homme, sa ou ses femmes, ainsi que ses enfants. Les femmes pratiquent surtout la vente au détail alors que l'homme pratique la vente de gros. Les enfants, principalement ceux qui ne veulent pas apprendre d'autres métiers, exercent l'activité avec leurs parents. Dès qu'ils deviennent majeurs, ils s'installent en indépendants mais maintiennent toujours les relations avec les parents, ce qui leur garantit l'accès au produit à travers le réseau familial (généralement à crédit). Certains grossistes sont aidés dans l'activité par un parent (frère, mère, nièce, bru ou même fille ou fils). Cette situation contraste avec celle observée dans les circuits du nord où 
l'activité n'a pas un ancrage familial à l'image des entreprises familiales du circuit fon. La seule exception a été observée à Copargo (Circuit dendi-tanéka) où le plus grand grossiste de cette localité mène l'activité avec sa femme et son fils. Le plus souvent, c'est la femme grossiste qui se fait parfois aider par sa fille ou son fils. Cependant l'expansion de la profession se fait parfois à travers le réseau familial et ceci dans les trois circuits en présence : une commerçante peut également initier une sœur qui va se lancer dans la profession. La logique qui entoure la vente à crédit, pose le problème des frontières, et de confiance entre les groupes ethniques en présence. La présente étude a mis en évidence, l'importance de la maîtrise de la langue de communication ${ }^{22}$ dans l'activité commerciale. Les grossistes Fon parviennent à écouler directement leurs marchandises alors que leurs homologues du nord passent principalement par leurs compatriotes basés dans le marché et qui maitrisent bien le fon. Mais nous avons vu également dans l'étude que la maîtrise de la langue fon seule ne suffit pas. Il faudra aussi maîtriser les circuits de distribution.

Il n'y a pas d'affrontement ou de rivalité entre les différents circuits en présence dans le marché. A aucun niveau, il n'a été observé le développement d'une stratégie collective visant à affaiblir ou à anéantir des circuits concurrents. Cette situation pourrait s'expliquer par l'inexistence d'une coordination des actions au niveau des différents circuits. Au sein de chaque circuit, il y a une certaine compétition entre les différents acteurs en ce qui concerne la conquête du gain de productivité. Cette compétition au niveau des circuits du nord est moins frappante simplement parce que la plupart du temps, les grossistes de ces circuits n'assurent pas eux-mêmes l'écoulement de leur marchandise dans le marché.

Selon le cas, la marchandise est confiée aux magasiniers ou aux demi-grossistes. Par contre, au niveau du circuit fon, les grossistes assurent directement l'écoulement du produit dans le marché. Chacun d'eux dispose d'un réseau aussi bien en amont (zones de production) qu'en aval (centres de consommation). Ces différents réseaux sont en compétition permanente aussi bien pour la conquête des aires de production que pour la conquête des parts de marché.

Les demi-grossistes du circuit dendi-tanéka sont à la fois dans une situation d'oligopole et d'oligopsone. Le même phénomène a été observé dans le circuit bariba-tchabè lors de la commercialisation des ignames précoces. Dans le premier cas, l'accès direct au produit n'est pas possible aux détaillantes des autres circuits alors que dans le second cas, il y a plus d'ouverture. Les détaillantes Fon par exemple peuvent s'approvisionner en igname auprès des grossistes du circuit bariba-tchabè. Enfin, la présente étude a mis en évidence, l'importance du commerce vivrier dans la dynamisation des relations ville-campagne.

\section{BIBLIOGRAPHIE}

Adanguidi J. 1999. "Formes de coordination au sein de la filière igname au Bénin". Etude d'un réseau de commercialisation, Working papers, 35, Berlin : Das arabische Buch. 
Godard X. 1985. "Quel est le rôle des transports dans la couverture des besoins alimentaires des villes ? Premières réflexions partielles". In AL TRESIAL-CERED-ORSTOM (éd.). Nourrir les villes en Afrique sub-saharienne. Paris : L'Harmattan.

Olivier de Sardan J.P. 1997. Anthropologie et développement. Paris : Karthala.

\section{NOTES}

1.Cet article représente un chapitre de notre dissertation sur le thème Sociologie de la filière igname au Bénin (Université de Hohenheim, Institut d'Economie et de Sociologie Rurales des pays tropicaux et subtropicaux. Stuttgart, Germany). Ce travail est supervisé par le Prof. Dr. Thomas Bierschenk (Johannes Gutenberg University). Institut for Ethnology and African Studies. Departement of Modem African Studies. 0-55099 Mainz, Germany. E-mail : biersche@mail.uni-mainz.de).

2.Il n'y a pas au niveau de ce circuit une spécialisation des commerçants par produit. Le même commerçant qui achète l'igname fraîche dans les villages achète aussi les cossettes d'igname. La priorité donnée à l'un ou l'autre des produits dépend de sa disponibilité et de l'époque de l'année considérée.

3.La vente se fait à Dantokpa, le grand marché international de Cotonou.

4.Ceux-là achètent une à deux tonnes et revendent par panier au prix courant de 11000 FCFA.

5.Société de Gestion des Marchés.

6.Comme nous l'avions déjà souligné plus haut, la vente de gros se fait ici par sac et par gros tas.

7.Il s'agit de la première récolte des ignames précoces, qui sortent déjà en juillet-août, et qui restent sur le marché jusqu'en octobre.

8.Le poids du sac d'igname fraîche varie entre 80 et $90 \mathrm{~kg}$.

9.Les hommes sont rares dans la profession. Une commerçante empêchée peut envoyer son fils à Cotonou avec la marchandise.

10.Il s'agit surtout de celles qui ont les moyens financiers. Car comme elles le disent, il faudrait avoir un important capital pour combiner les deux spéculations.

11.Comme nous l'avions déjà mentionné dans la partie consacrée à la mise en marché de l'igname au niveau de ce circuit, il n'y a pas, du moins dans la région d'étude, une production commerciale de cossettes d'igname comme c'est le cas dans la région de Tchaourou. Le plus souvent. c'est les femmes qui regroupent les écarts de cuisine et les transforment en cossettes d'igname qu'elles vendent dans les marchés ruraux. Le revenu sert généralement à l'achat des nécessaires de cuisine (condiments, pétrole. etc.).

12.A Djougou il y a des commerçants qui font le commerce de l'igname en direction de Cotonou et d'autres en direction de Malanville. Beaucoup de commerçants de Djougou vont se ravitailler à Copargo.

13. Une seule commercante de Copargo fait le commerce de l'igname en direction de Malanville via Kérou et Banikoara. Elle amène aussi la moutarde (de Néré). Au retour, elle achète des choses (pagnes, pommades, boissons) qu'elle revient vendre à Copargo. Certains commerçants font le commerce de l'igname en direction du Togo (marché frontalier d'Akra) au cours du mois de mai.

14.Ignames précoces. 
15.La durée de conservation peut atteindre quatre mois et même plus, ce qui est un record en la matière.

16.L'igname vendue par les commerçants de Copargo coûte un peu moins cher que celle vendue par ceux de Djougou. L'explication de cette situation se trouve dans le fait que les commerçants de Copargo vont se ravitailler dans les fermes de leur localité. Or ceux de Djougou vont se ravitailler à Copargo auprès des collecteurs locaux.

17. C'est seulement l'arachide, le maïs et les cossettes d'igname que ces grossistes confient aux magasiniers. Mais il s'agit de magasins différents de ceux utilisés par les commerçants du circuit bariba-tchabè.

18.Le terme cliente qui reviendra plusieurs fois dans la suite du texte, désigne la demi-grossiste.

19.Il s'agit ici des sacs plus gros que ceux utilisés par les grossistes du circuit bariba-tchabè.

20.Il s'agit des tas de six petits tubercules ou de trois gros tubercules. La revendeuse vend les tas tels qu'elle les a achetés.

21.La plupart de ceux-ci ne sont pas instruits.

22.Le fon est la langue de communication du marché Dantokpa.

\section{AUTEUR}

\section{JEAN ADANGUIDI}

Johannes Gutenberg University. Institut for Ethnology and African Studies. Departement of Modem Atrican Studies. 0-55099 Maint., Germany. Tel : +49-(0) 6131 392279H - adanguid@mail.uni-mainz.de 